\title{
Substitution de gypse de plâtre par des pourcentages de poudre de marbre
}

\author{
Abdellah HAOUAT and Omar AZZOUZ \\ ${ }^{1}$ Ingénieur doctorant en génie civil. Faculté des Sciences Oujda. Spécialité : Géo matériaux. Laboratoire des géosciences appliqués \\ ${ }^{2}$ Professeur d'enseignement supérieur. Faculté des Sciences Oujda. Spécialité : Géo matériaux. Laboratoire des géosciences \\ appliqués
}

Résumé : L'étude a pour but de vérifier La faisabilité d'ajouter de la poudre de marbre dans le gypse de plâtre en mélangeant les composants remplaçant diverses proportions de gypse par de la poudre de marbre pour évaluer le comportement mécanique du plâtre et en effectuant les tests suivants :

1. Consistance (test de la table d'écoulement eau / plâtre).

2. Résistance à la flexion.

3. Résistance à la compression.

4. Détermination adhésion au substrat.

Mots-clés : Béton, développement durable, ciment, l'environnement, poudre.

Abstract: The aim of the study is to verify the feasibility of adding marble powder to the gypsum plaster by mixing the components replacing various proportions of gypsum with marble powder to evaluate the mechanical behavior of the plaster and performing the tests. following:

1. Consistency (test of the water / plaster flow table).

2. Flexural strength.

3. Resistance to compression.

4. Determination of adhesion to the substrate.

Keywords: concrete, sustainable development, cement, the environment, powder.

\section{1-Définition :}

Le terme plâtre peut désigner le plâtre de gypse. Le plâtre est un matériau de construction similaire au mortier de ciment. Comme ces matériaux, le plâtre se présente sous forme de poudre sèche une fois mélangée à l'eau elle forme une pâte qui libère de la chaleur, puis durcit. Contrairement au mortier et au ciment, le plâtre reste assez mou après la prise et peut être facilement manipulé avec des outils en métal ou même du papier de verre. Ces caractéristiques font que le plâtre convient à la finition plutôt qu'au matériau porteur.

\section{$\underline{\text { 2-Processus de production de plâtre }}$}

Le gypse est converti en plâtre en éliminant une partie des substances chimiques eau combinée. Chauffer le gypse pendant une heure donne un hémihydrate, avec trois quarts de l'eau enlevés. 
Un chauffage prolongé sur plusieurs heures entraîne la formation d'anhydrite avec pratiquement aucune des eaux chimiquement combinées restantes.

L'anhydrite durcit plus lentement et est un plâtre légèrement plus résistant que l'hémihydrate, mais avec l'inconvénient du coût de production ajouté.

En pratique, un système de production simple serait le plus probablement a donner un mélange des phases hémihydrate et anhydrite. Le plâtre de gypse par sa combinaison chimique avec de l'eau afin former du sulfate de calcium dihydraté solide.

Un type spécial de plâtre, appelé alpha hémi-hydrate, peut être produit en faisant réagir le brut matériel sous vapeur à haute pression dans des conditions strictement contrôlées. Ce plâtre durcit est utilisé dans les travaux dentaires et comme un plâtre de modélisation, mais est impossible à produire sans matériel de production coûteux.

Dans les détails, le plâtre est obtenu en chauffant du gypse naturel à $128^{\circ} \mathrm{C}$, ce qui lui confère un constituant essentiel, le sulfate de calcium dihydraté, perd une demi-molécule de cristallisation ainsi la transformation en hémihydrate de gypse.

La formule chimique est CaSO4 • 1/2 H2O est également connu sous le nom de plâtre de Paris, car le gypse des carrières du quartier de Montmartre à Paris a longtemps fourni du gypse brûlé utilisé à des fins diverses. Une grande partie du plâtre commercial produit industriellement est du plâtre de paris. Il a une densité de $24,52-26,49 \mathrm{~N} / \mathrm{dm} 3$ et contient $5-7 \%$ d'eau.

Pulvérisé et mélangé avec de l'eau, absorbe facilement l'eau perdue et forme un ensemble compact d'agrégats cristaux.

Ainsi, le plâtre de Paris est le sulfate de calcium hémihydraté, il est créé en chauffant du gypse environ $150{ }^{\circ} \mathrm{C}$, comme le montrent les réactions chimiques suivantes (Turco, 1996):

$\mathrm{CaSO} 4 \cdot 2 \mathrm{H} 2 \mathrm{O} \rightarrow \mathrm{CaSO} 4 \cdot 1 / 2 \mathrm{H} 2 \mathrm{O}+1 \frac{1}{2} \mathrm{H} 2 \mathrm{O}$ (libéré sous forme de vapeur)

Le plâtre résultant durcira en quelques minutes après l'ajout d'eau, une partie qui est recombiné chimiquement, de sorte que la composition de l'enduit de plâtre est identique à celle du gypse d'origine. Lorsque la poudre de plâtre sèche est mélangée avec un chauffage supplémentaire à $163^{\circ} \mathrm{C}$ (en pratique, être atteint ou dépassé $200^{\circ} \mathrm{C}$ ), le sulfate calcium hémihydrate obtenu d'abord à $128^{\circ} \mathrm{C}$ perd toute l'eau de cristallisation et devient sulfate de calcium anhydre, soluble, de formule chimique CaSO4. Cela conduit au soluble anhydrite ou anhydrite $\alpha$, avec un poids spécifique de 23,94 - 24,03 N / dm3 et un pincement plus lent, est également connu sous le nom d'usine de plâtre ou de plâtre ordinaire ((plaster of Paris in english; yeso de costrucción in 
Spanish). Le comportement du plâtre commun entre $128^{\circ} \mathrm{C}$ et $163^{\circ} \mathrm{C}$ peut être schématisé dans les réactions chimiques suivantes (Turco, 1996).

$\mathrm{CaSO} 4 \cdot 1 / 2 \mathrm{H} 2 \mathrm{O} \rightarrow \mathrm{CaSO} 4+\frac{1}{2} \mathrm{H} 2 \mathrm{O}$ (libéré sous forme de vapeur)

Les deux processus sont réversibles.

\section{3- Phase expérimentale}

L'étude a pour but de vérifier La faisabilité d'ajouter de la poudre de marbre dans le plâtre a été étudiée en mélangeant les composants remplaçant diverses proportions de gypse par de la poudre de marbre et en effectuant les tests suivants pour évaluer le comportement mécanique : consistance (test de la table d'écoulement eau / plâtre), résistance à la flexion, résistance à la compression et détermination adhésion au substrat.

\section{3-1 Matériel et méthodes}

La recherche s'est concentrée sur la possibilité d'ajouter de la poudre de marbre au gypse pour la fabrication de plâtre, en particulier de plâtre à projection de plâtre qui est un plâtre de gypse formulé pour application mécanique, mélangé avec de l'eau jusqu'à la consistance requise et appliqué par la machine de projection à l'arrière-plan.

Pour cette partie expérimentale, on a préparé différents mélanges obtenus en remplaçant divers proportions de gypse avec de la poudre de marbre et ont été effectuées les tests suivants, conformément aux normes nationales indiquées dans la norme UNI EN 13279-2: 2004 - «Plâtre liants et enduits de plâtre », afin d'évaluer le comportement mécanique du composés créés:

1. détermination du rapport eau / plâtre par la méthode de la table de flux;

2. détermination de la résistance à la flexion;

3. détermination de la résistance à la compression;

4. détermination de l'adhésion.

Deux types de matériaux différents ont été utilisés dans cette recherche: le gypse et la poudre de marbre comme agrégat.

\section{3-1-1 Détermination du rapport eau / plâtre avec la méthode de la table de flux}

Pour définir cette phase expérimentale, la première étape a été d'identifier le rapport eau / plâtre et nous l'avons fait avec la méthode de la table de flux et pour tous les plans de mixage créés.

Cette méthode est utilisée pour les emplâtres de gypse prémélangés. En règle générale, le rapport eau / plâtre est déterminé par la méthode d'essais et d'erreurs jusqu'à la formation d'une couche d'un diamètre spécifié, lorsqu'un cône tronqué, rempli de la boue, est enlevé et secoué de la manière décrite au-dessous de. 
Le rapport eau / plâtre pour le plâtre de gypse prémélangé est défini par une cohérence. La cohérence requise est atteinte lorsque la détermination empirique du diamètre de la couche de plâtre est de $(165 \pm 5) \mathrm{mm}$.

Nous avons donc poursuivi cette procédure pour tous les plans de mixage créés, qui sont listés dans le tableau 6.2.

Tout d'abord, $500 \mathrm{~g}$ de plâtre de gypse ont été pesés à $1 \mathrm{~g}$. La quantité d'eau déterminée par un rapport eau / plâtre de 0,6 a été pesé dans le bol de mélange sec.

Ensuite, la procédure consistait à plier le plâtre de gypse dans l'eau et à mélanger préalablement avec la spatule et la palette à la main pendant environ 1 min. Le plâtre a ensuite été mélangé avec le mélangeur et la pagaie pendant 1 min à basse vitesse $(140 \pm 5) \min -1$ rotation et $(62 \pm 5)$ min - un mouvement planétaire.

Le cône d'affaissement a été placé au centre de la plaque de la table de flux et il a été maintenu fermement en position avec une main (Figure 7.1). L'excès de plâtre a été versé dans le cône avec une spatule.

\section{Figure 1 : Méthode du table de flux.}

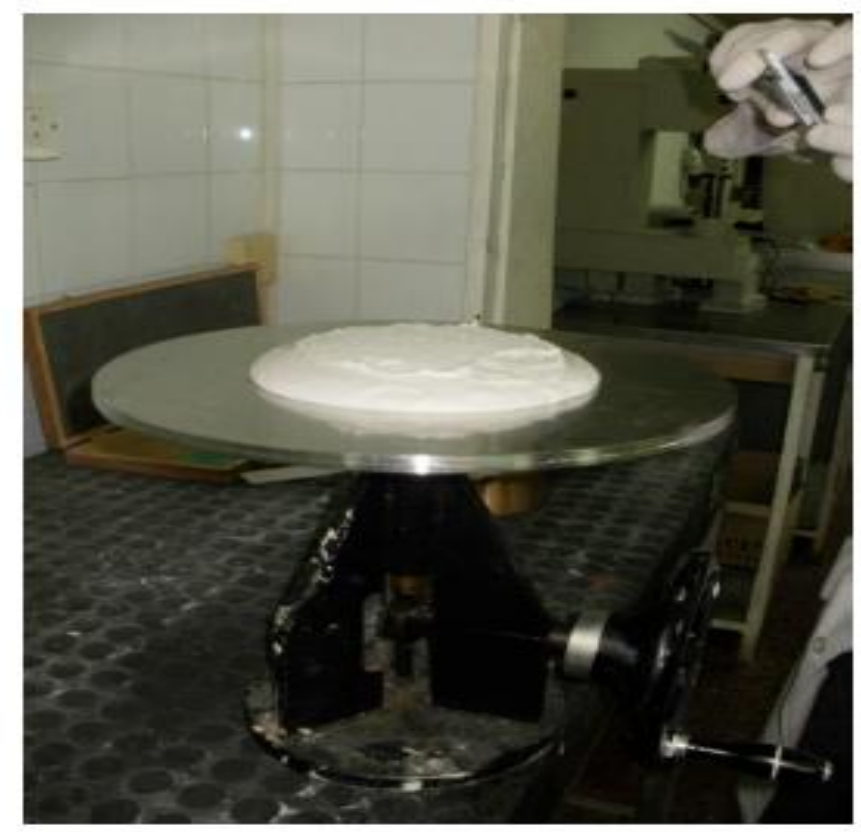

\section{Source : propre}

Le rapport eau / plâtre R est donné par la formule 1.

$\mathrm{R}=\mathrm{m} 2 / \mathrm{m} 1(1)$

où:

m2 est la masse d'eau de mélange, en g;

m1 est la masse de plâtre de gypse, en g. 
Et les résultats finaux sont reportés dans le tableau ci-dessous.

Tableau 1: Modèles de mélanges pour l'expérimentation sur les plâtres et rapport entre le rapport eau / plâtre.

\begin{tabular}{|c|c|c|c|}
\hline Mix Design & Gypsum wt. \% & Marble dust wt. \% & Water/Plaster ratio \\
\hline $\mathbf{1}$ & 100 & 0 & 0.60 \\
\hline $\mathbf{2}$ & 95 & 5 & 0.60 \\
\hline $\mathbf{3}$ & 90 & 10 & 0.57 \\
\hline $\mathbf{4}$ & 85 & 15 & 0.55 \\
\hline
\end{tabular}

Source : propre

\section{3-1.2 Détermination de la résistance à la flexion}

\section{3-1-2-1 Expression des résultats}

La résistance à la flexion $\mathrm{PF}$ est donnée par la formule 2:

$\mathrm{PF}=0,00234 \times \mathrm{P}(2)$

où:

$\mathrm{PF}$ est la résistance à la flexion, en $\mathrm{N} / \mathrm{mm} 2$;

$\mathrm{P}$ est la charge de rupture moyenne en $\mathrm{N}$ d'au moins trois valeurs obtenues.

À la fin, les valeurs moyennes obtenues pour la rupture lors des essais de résistance à la flexion sont indiquées dans les tableaux 6.3, 6.4 et dans la figure 6.3

Tableau 2: Données obtenues pour les essais de rupture en résistance à la flexion.

\begin{tabular}{|l|c|c|c|c|}
\hline \multicolumn{5}{|c|}{ BREAKING LOAD, P [kN] } \\
\cline { 1 - 4 } Mix Design & $\mathbf{1}$ & $\mathbf{2}$ & $\mathbf{3}$ & Mean Value \\
\cline { 1 - 4 } MP0 - 1 & 0.63 & 0.64 & 0.65 & 0.56 \\
\hline MP0 - 2 & 0.54 & 0.45 & 0.47 & 0.61 \\
\hline MP5 & 0.65 & 0.55 & 0.63 & 0.64 \\
\hline MP10 & 0.60 & 0.68 & 0.65 & 0.72 \\
\hline MP15 & 0.77 & 0.70 & 0.69 & \\
\hline
\end{tabular}

Source : propre 
Tableau 3: Données obtenues pour les essais de rupture en résistance à la flexion.

\begin{tabular}{|l|c|c|c|c|}
\hline Mix Design & $\begin{array}{c}\text { Test specimen 1 } \\
\boldsymbol{P}_{\boldsymbol{F}}\left[\mathbf{N} / \mathbf{m m}^{2}\right]\end{array}$ & $\begin{array}{c}\text { Test specimen 2 } \\
\boldsymbol{P}_{\boldsymbol{F}}\left[\mathbf{N} / \mathbf{m m}^{2}\right]\end{array}$ & $\begin{array}{c}\text { Test specimen 3 } \\
\boldsymbol{P}_{\boldsymbol{F}}\left[\mathbf{N} / \mathbf{m m}^{2}\right]\end{array}$ & $\begin{array}{c}\text { Mean Value } \\
\boldsymbol{P}_{\boldsymbol{F}}\left[\mathbf{N} / \mathbf{m m}^{2}\right]\end{array}$ \\
\cline { 1 - 3 } MP0 - 1 & 1.47 & 1.50 & 1.52 & 1.32 \\
\cline { 1 - 3 } MP0 - 2 & 1.26 & 1.05 & 1.10 & 1.43 \\
\hline MP5 & 1.52 & 1.29 & 1.47 & 1.51 \\
\hline MP10 & 1.40 & 1.59 & 1.52 & 1.68 \\
\hline
\end{tabular}

Source : propre

\section{3-1-3 Détermination de la résistance à la compression}

\section{3-1-3-1 Expression des résultats}

Les valeurs moyennes des valeurs d'essai ont été calculées et exprimées en $\mathrm{N} / \mathrm{mm}^{2}$ (tableau 4 et tableau 5). La résistance à la compression Rc est calculée par la formule 3.

$$
\mathrm{Rc}=\mathrm{Fc} / 1600
$$

où

Rc est la résistance à la compression, en $\mathrm{N} / \mathrm{mm} 2$;

Fc est la charge maximale à la rupture, en $\mathrm{N}$;

1600 (40 mm x $40 \mathrm{~mm}$ ) est l'aire des plateaux en mm2.

Tableau 4: Données obtenues pour les essais de résistance à la rupture.

\begin{tabular}{|l|c|c|c|c|c|c|c|}
\hline \multicolumn{7}{|c|}{ MAXIMUM LOAD AT FRACTURE, P [kN] } \\
\cline { 1 - 6 } Mix Design & $\mathbf{1 - 1}$ & $\mathbf{1 - 2}$ & $\mathbf{2 - 1}$ & $\mathbf{2 - 2}$ & $\mathbf{3 - 1}$ & $\mathbf{3 - 2}$ & Mean Value \\
\hline MP0 - 1 & 6.65 & 6.70 & 6.84 & 6.89 & 7.20 & 6.99 & \multirow{2}{*}{6.50} \\
\cline { 1 - 2 } - 2 & 6.14 & 6.02 & 6.14 & 6.07 & 6.21 & 6.11 & \\
\hline MP5 & 6.49 & 6.15 & 6.41 & 6.65 & 6.99 & 6.60 & 6.55 \\
\hline MP10 & 7.28 & 7.89 & 7.86 & 7.70 & 7.39 & 7.74 & 7.64 \\
\hline MP15 & 7.73 & 7.02 & 7.61 & 8.06 & 6.74 & 6.85 & 7.34 \\
\hline
\end{tabular}

$\underline{\text { Source : propre }}$ 
Tableau 5: Résultats obtenus pour les essais de rupture en résistance à la compression.

\begin{tabular}{|c|c|c|c|c|c|c|c|}
\hline \multirow[t]{2}{*}{ Mix Design } & \multicolumn{2}{|c|}{$\begin{array}{c}\text { Test specimen } \\
1 \\
R_{c}\left[\mathrm{~N} / \mathrm{mm}^{2}\right]\end{array}$} & \multicolumn{2}{|c|}{$\begin{array}{c}\text { Test specimen } \\
2 \\
R_{c}\left[\mathrm{~N} / \mathrm{mm}^{2}\right]\end{array}$} & \multicolumn{2}{|c|}{$\begin{array}{c}\text { Test specimen } \\
3 \\
R_{c}\left[\mathrm{~N} / \mathrm{mm}^{2}\right]\end{array}$} & \multirow[t]{2}{*}{$\begin{array}{l}\text { Mean Value } \\
R_{c}\left[\mathrm{~N} / \mathrm{mm}^{2}\right]\end{array}$} \\
\hline & $1-1$ & $1-2$ & $2-1$ & $2-2$ & 3-1 & 3-2 & \\
\hline MP0 - 1 & 4.16 & 4.19 & 4.28 & 4.31 & 4.50 & 4.37 & \multirow{2}{*}{4.06} \\
\hline MP0 - 2 & 3.84 & 3.76 & 3.84 & 3.79 & 3.88 & 3.82 & \\
\hline MP5 & 4.06 & 3.84 & 4.01 & 4.16 & 4.37 & 4.13 & 4.09 \\
\hline MP10 & 4.55 & 4.93 & 4.91 & 4.81 & 4.62 & 4.84 & 4.78 \\
\hline MP15 & 4.83 & 4.39 & 4.76 & 5.04 & 4.21 & 4.28 & 4.58 \\
\hline
\end{tabular}
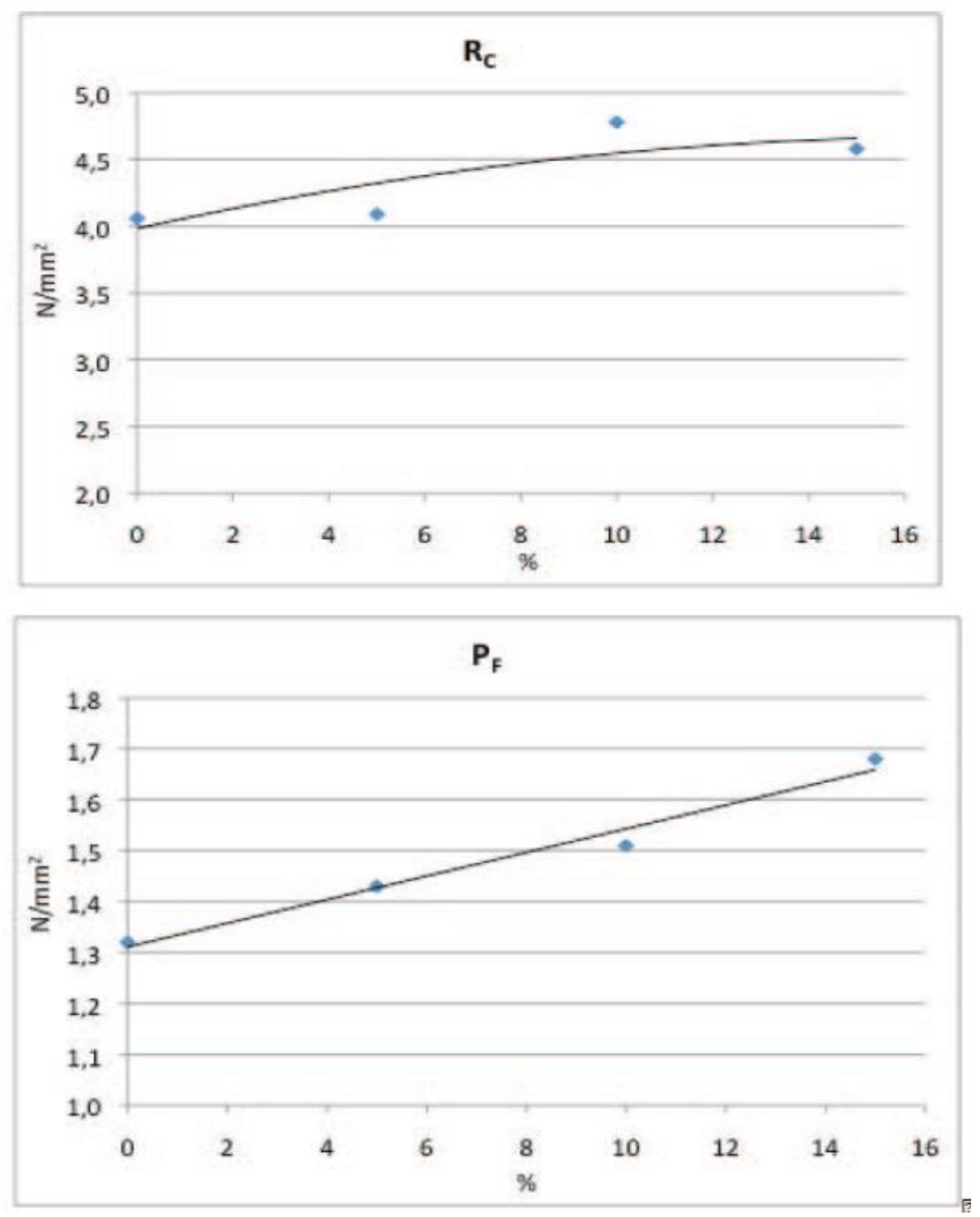

Figure 3: Marche de résistance à la compression 


\section{3-1-4 Détermination de l'adhérence}

\section{3-1-4-1 Expression des résultats}

La force d'adhérence individuelle a été calculée par la formule 6.4:

$\mathrm{Ru}=\mathrm{Fu} / \mathrm{A}$

où

$\mathrm{Ru}$ est la force d'adhérence en $\mathrm{N} / \mathrm{mm} 2$;

Fu est la charge de rupture en $\mathrm{N}$;

A est la zone de test de l'éprouvette cylindrique, en mm2.

La force d'adhérence a été calculée comme la valeur moyenne à partir des valeurs individuelles de l' échantillon à $0,01 \mathrm{~N} / \mathrm{mm} 2$ près.

Les résultats finaux sont reportés dans les tableaux suivants.

Tableau 6 : Données obtenues pour les tests d'adhésion.

\begin{tabular}{|l|c|c|c|}
\hline \multirow{4}{*}{ Mix Design } & $\begin{array}{c}\text { Test specimen } \\
\boldsymbol{R}_{u}\left[\mathbf{N} / \mathbf{m m}^{2}\right]\end{array}$ & $\begin{array}{c}\text { Test specimen 2 } \\
\boldsymbol{R}_{u}\left[\mathbf{N} / \mathbf{m m}^{2}\right]\end{array}$ & $\begin{array}{c}\text { Test specimen 3 } \\
\boldsymbol{R}_{u}\left[\mathbf{N} / \mathbf{m m}^{2}\right]\end{array}$ \\
\cline { 2 - 4 } & 0.19 & 0.17 & 0.29 \\
\cline { 2 - 4 } & Plaster & Interface & Interface \\
\cline { 2 - 4 } MP5 & 0.19 & 0.23 & 0.15 \\
\cline { 2 - 5 } & Interface & Interface & Plaster \\
\cline { 2 - 5 } MP10 & 0.19 & 0.20 & 0.23 \\
\cline { 2 - 5 } & Interface & Interface & Plaster \\
\cline { 2 - 5 } & O.17 & O.18 & Interface \\
\hline \multirow{2}{*}{ MP15 } & Interface & Interface &
\end{tabular}

Source : propre

\section{3-2 Résultats et discussion}

\section{3-2-1 Résistance à la flexion}

On peut constater que la poudre de marbre a une relation directe avec la résistance à la flexion du plâtre de gypse (tableau 2 et figure 3). En fait, l'utilisation de poudre de marbre a entraîné une augmentation de la résistance à la flexion, la valeur moyenne passant de 1,32 [N / mm2], spécimens de contrôle MP0, à 1,68 [N/ $\mathrm{mm} 2], \mathrm{MP} 15$. 


\section{3-2-2 Résistance à la compression}

L'effet de la poussière de marbre sur la résistance à la compression des enduits de gypse durcis et durcis à différents pourcentages de substitution est présenté dans le tableau 6.6 et la figure 6.4. Les données montrent qu'avec $10 \%$ d'ajout de poudre de marbre, on a généralement atteint le maximum de résistance, ce qui montre clairement qu'en termes de résistance à la compression, la poudre de marbre peut améliorer la résistance des enduits.

\section{3-2-3 Test d'adhésif}

Lors des tests effectués sur la résistance adhésive, il était impossible de calculer les valeurs moyennes pour les différentes valeurs obtenues, en raison des ruptures survenues.

\section{4- CONCLUSION}

Comme expliqué précédemment et selon les résultats du test, la poudre de marbre augmente considérablement la résistance à la flexion et à la compression. Une perte de résistance à la compression a été observée dans le cas de l'application de $15 \%$ de poudre de marbre, mais la valeur reste considérablement supérieure à celle de l'échantillon de base.

Il a été constaté que les tests d'adhésif peuvent ne pas être suffisants pour la détermination de la force d'adhérence, en raison des fausses ruptures. Les causes liées aux problèmes de matériaux sont liées à l'ensemble des caractéristiques mécaniques, physiques et chimiques. Par conséquent, l'amélioration des propriétés est le seul moyen de garantir leur bonne exécution et leur bon fonctionnement lorsqu'elles sont appliquées dans le bâtiment.

En conclusion, les résultats ont démontré que la poudre de marbre, ajoutée à la poudre de plâtre avant le calibrage, modifie certaines propriétés physiques du liant après le durcissement, par exemple la résistance à la compression et à la flexion.

\section{BIBLIOGRAPHIE}

[1] Almeida N., Branco F., Santos J.R., (2007). Recycling of stone slurry in industrial activities: Application to concrete misture. Building and Environment 42(2007): 810 - 819.

[2] Binici, H., Kaplan, H., Y1lmaz, S., 2007, "Influence of marble and limestone dusts as additives on some mechanical properties of concrete”.In: ScientificResearch and Essay Vol.2 (9), 372-379, September 2007.

[3] Bontoux, L., Leone, F., 1997, “The Legal Definition of Waste and its Impact on Waste Management in Europe”. Office for Official Publications of the European Communities, EUR 17716 EN, November, Luxembourg.

[4] European Council Decision (2001/573/EC), Council Decision of 23 July 2001 amending Council Decision 2000/532/EC as regards the list of wastes, Official Journal of the European Communities: L 203/18, Brussels, July 2001

[5] Gertsakis, J., Lewis, H., 2003, "Sustainability and the Waste Management Hierarchy”.A discussion paper for EcoRecycle Victoria, RMIT University, Melbourne

[6] Marras, G., Careddu, N., Internicola, C., Siotto, G., 2010. "Recovery and reuse of marble powder by-product". In: Proceedings of the Global Stone Congress 2010. 02 - 05 March 2010, Alicante (Spain).

[7] OECD (1998) Waste Management Policy Group.Final Guidance Document for Distinguishing Waste from Non-waste. ENV/EPOC/WMP(8)1/REV1. Paris, 23-24 April 1998. 
[8] OSNET Editions vol. 7, "Optimising quarrying techniques and practices”, edited by N. Terezopoulos, I. Paspaliaris, Athens 2004.

[9] OSNET Editions vol. 12, "Environmental friendly practices for natural stone exploitation”, edited by A. Dieb, N. Bonito, I. Paspaliaris, Athens 2004.

[10] Pongracz, E., 2002, "Re-defining the concepts of waste and waste management - Evolving the theory of waste management", Oulu University Press, ISBN 951-42-6821-0.

[11] Poncrácz, E., Philips, PS., Keiski, R1., 2004, "From waste minimization to resources use optimization: Definitions and legislative background”. In: Pongrácz E. (ed.) Proceedings of the Waste Minimisation and Resources Use Optimisation Conference. June 10th 2004, University of Oulu.Oulu University Press.

[12] "Report on Special Waste Management in Sardinia - Analysis of MUD 2005 data" published by the Environmental Protection Department of the Autonomous Region of Sardinia (April 2007).

[13] Segadães, A.M., Carvalho, M.A., Acchar, W., Appl. Clay Sci. 30 (2005) 42-52.

[14] SiddarthPareek, 2001. "Gainful utilization of Marble waste. An effort towards protection of ecology and environment", and http.//www.cdos- india.com/papers. 